\section{(2) OPEN ACCESS}

\title{
Influence of sex and phenotype on cardiac outcomes in patients with Fabry disease
}

\author{
Mohamed El Sayed 다, ${ }^{1}$ Alexander Hirsch 이, ${ }^{2,3}$ Matthijs Boekholdt, ${ }^{4}$ \\ Laura van Dussen, ${ }^{1}$ Mareen Datema, ${ }^{1}$ Carla Hollak, ${ }^{1}$ Mirjam Langeveld ${ }^{1}$
}

\begin{abstract}
- Prepublication history and additional material are published online only. To view, please visit the journal online (http://dx.doi.org/10.1136/ heartinl-2020-317922)
\end{abstract}

${ }^{1}$ Endocrinology and Metabolism, Amsterdam UMC Locatie AMC Amsterdam, The Netherlands ${ }^{2}$ Cardiology, Erasmus Medical Center, Rotterdam, ZuidHolland, The Netherlands ${ }^{3}$ Radiology and Nuclear Medicine, Erasmus Medical Center, Rotterdam, ZuidHolland, The Netherlands ${ }^{4}$ Cardiology, Amsterdam UMC Locatie AMC, Amsterdam, Noord Holland, The Netherlands

\section{Correspondence to} Dr Mirjam Langeveld, Endocrinology and Metabolism, Amsterdam UMC Locatie AMC, Amsterdam 1100 DD, The Netherlands;

m.langeveld@amsterdamumc.n

Received 29 July 2020

Revised 5 January 2021

Accepted 13 January 2021

Published Online First

10 February 2021
ABSTRACT

Objective This study describes the influence of sex and disease phenotype on the occurrence of cardiac events in Fabry disease (FD).

Methods Cardiac events from birth to last visit (median age 50 years) were recorded for 213 patients with FD.

Patients were categorised as follows : men with classical FD $(n=57)$, men with non-classical FD $(n=26)$, women with classical FD $(n=98)$ and women with non-classical FD $(n=32)$, based on the presence of classical FD symptoms, family history (men and women), biomarkers and residual enzyme activity (men). Event rates per 1000 patient-years after the age of 15 years and median event-free survival (EVS) age were presented. Influence of disease phenotype, sex and their interaction was studied using Firth's penalised Cox regression.

Results The event rates of major cardiovascular events (combined endpoint cardiovascular death (CVD), heart failure (HF) hospitalisation, sustained ventricular arrhythmias (SVAs) and myocardial infarction) were 11.0 (95\% Cl 6.6 to 17.3) in men with classical FD (EVS 55 years), 4.4 (95\% Cl 2.5 to 7.1$)$ in women with classical FD (EVS 70 years) and 5.9 (95\% Cl 2.6 to 11.6) in men with non-classical FD (EVS 70 years). None of these events occurred in women with non-classical FD. Sex and phenotype significantly influenced the risk of major adverse cardiovascular event. CVD was the leading cause of death $(75 \%)$ to which HF contributed most (42\%). The overall rate of SVA was low (14 events in nine patients $(4 \%))$.

Conclusions Sex and phenotype greatly influence the risk and age of onset of cardiac events in FD. This indicates the need for patient group-specific follow-up and treatment.

\section{INTRODUCTION}

Fabry disease (FD) is a rare X-linked lysosomal storage disease that is caused by mutations in the Galactosidase alpha gene, resulting in reduced alpha-galactosidase A enzyme activity. ${ }^{1} 2$ Accumulation of the enzyme's substrate globotriaosylceramide (Gb3) and its derivatives is the primary trigger for damage and dysfunction of various tissues and organs, including vascular endothelium and the heart. ${ }^{3-5}$

Due to the X-linked mode of inheritance, men are generally more severely affected and disease manifestations occur earlier compared with women. In addition, a distinction is made between classical and non-classical disease phenotypes, with significant differences in onset and progression of symptoms, organ damage and outcome between these two groups. The classical form of FD in men is characterised by greatly reduced or absent alphagalactosidase A activity, resulting in manifestations in multiple organs, starting from late adolescence. ${ }^{67}$ Non-classical disease manifests itself later in adulthood and often affects only the heart. ${ }^{8-10}$

Early cardiac manifestations of FD are bradycardia, shortened PQ interval, low native T1 value on cardiac MRI and, in male patients and a subset of female patients, cardiac hypertrophy. ${ }^{11} 12$ As the disease progresses, conduction abnormalities (CAs), supraventricular arrhythmias, ischaemic heart disease, diastolic and systolic dysfunctions and ultimately overt heart failure (HF), leading to cardiac death, may occur. ${ }^{313-22}$ On the other hand, a significant number of patients will remain asymptomatic, even at an advanced age. ${ }^{7}$ Limited evidence is available on the risk and timing of cardiac manifestations and events in different patient groups (ie, men vs women, classical vs non-classical FD phenotype). ${ }^{3}{ }^{23}$ It is important to generate these data, as it will guide patient-specific follow-up and timing of treatment initiation, risk assessment (eg, for sudden cardiac death (SCD)) and evaluation of new FD therapies that are currently in various stages of development. To answer the open questions, we performed a retrospective study in an FD cohort under follow-up at the Amsterdam University Medical Centres (AUMC), which is unique in both its size, as well as the length of systematic follow-up of patients, providing detailed clinical data on cardiac outcome.

\section{METHODS}

\section{Patient and public involvement}

This is an observational longitudinal retrospective study, using data from all adult patients with a definite diagnosis of FD (figure 1) that have been under follow-up at any time at the AUMC, the national referral centre for patients with FD in the Netherlands (table 1).

\section{Data collection}

Patients were divided into four groups: men with classical FD, men with non-classical FD, women with classical FD and women with non-classical FD, based on the presence of classical FD symptoms (cornea verticillata, acroparesthesia or angiokeratoma), family history or known mutation-phenotype associations (men and women) and biomarkers and residual alpha-galactosidase A activity (men) ${ }^{724}$
To cite: El Sayed M, Hirsch A Boekholdt M, et al. Heart 


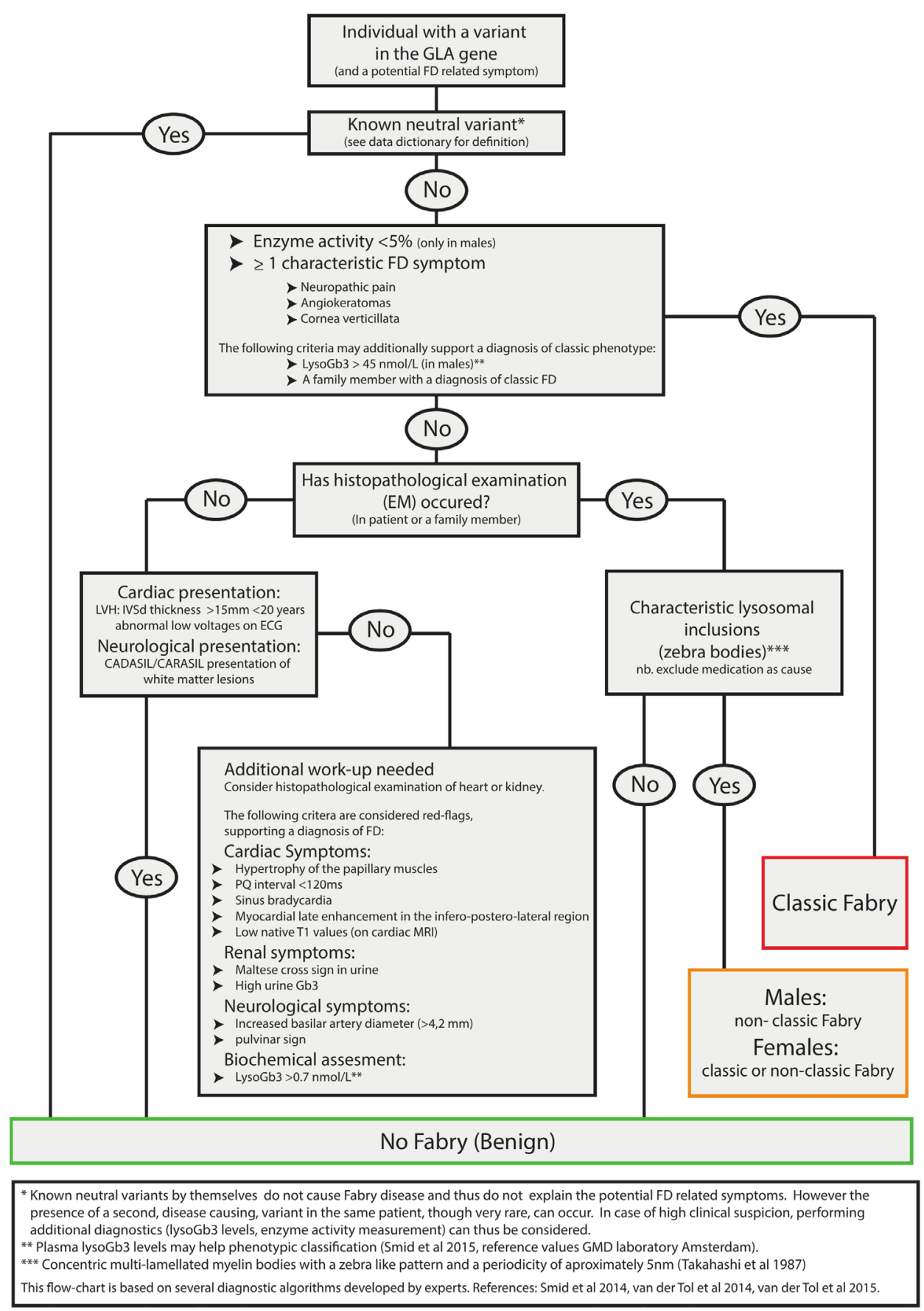

Figure 1 Flowchart for the diagnosis and phenotype allocation in FD. CADASIL, cerebral autosomal dominant arteriopathy with subcortical infarcts and leucoencephalopathy; CARASIL, cerebral autosomal recessive arteriopathy with subcortical infarcts and leucoencephalopathy; EM, electron microscopy; FD, Fabry disease; GLA, galactosidase alpha; IVSd, diastolic interventricular septum diameter.

(see figure 1 for details). Patients attended the clinic for standardised follow-up visits.

Between September 2018 and January 2019, all available patients charts, clinical letters, cardiac imaging reports from birth until the last outpatient clinic visit were investigated to record the following events: cardiovascular death (CVD), HF hospitalisation, sustained ventricular arrhythmias (SVA), myocardial infarction (MI), CA, pacemaker or implantable cardiac defibrillator (ICD) implantation, atrial fibrillation, coronary artery disease (CAD), percutaneous coronary intervention, coronary artery bypass graft surgery, systolic dysfunction on cardiac MRI or, if unavailable on echocardiography, left ventricular outflow tract (LVOT) obstruction, moderate or severe valve disease and other heart surgery/intervention. The combined endpoint major adverse cardiovascular event (MACE) was defined as the occurrence of at least one of the following events: CVD, HF hospitalisation, SVA or MI. SVA included SCD, sudden cardiac arrest, sustained ventricular tachycardia lasting $>30 \mathrm{~s}$, appropriate ICD shock, and ventricular fibrillation. CA were defined as a composite of seconddegree atrioventricular (AV) block type II, third-degree AV block, sinus arrest and device implantation for CA. Endpoint definitions are provided in online supplemental table 1). Events were adjudicated by a panel of experts consisting of 


\begin{tabular}{|c|c|c|c|c|c|}
\hline & & Men & & Women & \\
\hline & All & Classical & Non-classical & Classical & Non-classical \\
\hline General & & & & & \\
\hline Number of patients, $\mathrm{n}(\%)$ & $213(100)$ & $57(27)$ & $26(12)$ & $98(46)$ & $32(15)$ \\
\hline Age at last outpatient visit or death (years), median (range) & $50(19-83)$ & $45(19-66)$ & $64(26-78)$ & $51(19-83)$ & $47(23-79)$ \\
\hline Cumulative follow-up from the age of 15 (years) & 7090 & 1546 & 1190 & 3213 & 1140 \\
\hline Age at first outpatient clinic visit (years) median (range) & $42(3-77)$ & $30(3-58)$ & $60(22-70)$ & $41(7-71)$ & $44(19-77)$ \\
\hline Comorbidities & & & & & \\
\hline Number of patients with CVA at any time, $n(\%)$ & $25(12)$ & $9(16)$ & $5(19)$ & $11(11)$ & $0(0)$ \\
\hline Obesity, ${ }^{*} \mathrm{n}(\%)$ & $30 / 198(15)$ & $1 / 51(2)$ & $4 / 24(17)$ & $16 / 93(17 \%)$ & $9 / 30(30)$ \\
\hline Smoking, ${ }^{*} \mathrm{n}(\%)$ & $75 / 177(42)$ & $17 / 44(39)$ & $14 / 23(61)$ & $35 / 87(40)$ & $9 / 23(39)$ \\
\hline Hypertension, ${ }^{*} \mathrm{n}(\%)$ & $48 / 199(24)$ & $8 / 51(16)$ & $11 / 26(42)$ & 18/95 (19) & $11 / 27(41)$ \\
\hline Dyslipidaemia, ${ }^{*}$ (\%) & $14 / 187(8)$ & $0 / 43(0)$ & $7 / 23(30)$ & $5 / 91(6)$ & $2 / 30(7)$ \\
\hline Diabetes mellitus, ${ }^{*} \mathrm{n}(\%)$ & $4 / 203(2)$ & $0 / 52(0)$ & $2 / 25(8)$ & $1 / 96(1)$ & $1 / 30(3)$ \\
\hline Cardiac imaging & & & & & \\
\hline Echocardiography & & & & & \\
\hline Available echocardiography at any time, $n(\%)$ & $181(85)$ & $49(86)$ & $21(81)$ & $87(89)$ & $24(75)$ \\
\hline Left ventricular hypertrophy on echocardiography at any time, $n(\%) \dagger$ & $90 / 181(50)$ & $24 / 49(49)$ & $16 / 21(76)$ & $43 / 87(49)$ & $7 / 24(29)$ \\
\hline Cardiac MR $\ddagger$ & & & & & \\
\hline Available cardiac MR at last follow-up, n (\%) & $141(66)$ & $41(72)$ & $11(42)$ & $71(73)$ & $18(56)$ \\
\hline Left ventricular ejection fraction (\%), median (range) & $61(24-84)$ & $57(24-70)$ & $57(48-67)$ & $63(51-84)$ & $61(57-70)$ \\
\hline $\begin{array}{l}\text { Available cardiac MR, where myocardial fibrosis was assessed by late } \\
\text { gadolinium enhancement, } \mathrm{n}(\%)\end{array}$ & 139/141 (99) & $39 / 41(95)$ & $11 / 11(100)$ & $71 / 71(100)$ & $18 / 18(100)$ \\
\hline Myocardial fibrosis on cardiac MR, $\mathrm{n}(\%)$ & $54 / 139(39)$ & $13 / 39(33)$ & $6 / 11(55)$ & $29 / 71(41)$ & $6 / 18(33)$ \\
\hline ERT & & & & & \\
\hline Patients on ERT, $\mathrm{n}(\%)$ & $128(60)$ & $47(83)$ & $12(46)$ & $62(63)$ & $7(22)$ \\
\hline Age start ERT (years), median (range) & $42(10-77)$ & $27(10-58)$ & $59(20-68)$ & $46(16-71)$ & $60(30-77)$ \\
\hline Untreated patients, $\mathrm{n}(\%)$ & $85(40)$ & $10(17)$ & $14(54)$ & $36(37)$ & $25(78)$ \\
\hline Reasons for not starting ERT & & & & & \\
\hline $\begin{array}{l}\text { Diagnosis through family screening, absent or minimal organ } \\
\text { involvement, } \mathrm{n}(\%)\end{array}$ & $41(19)$ & $1(2)$ & $3(12)$ & $17(17)$ & $20(63)$ \\
\hline $\begin{array}{l}\text { Diagnosis not through family screening, absent or minimal organ } \\
\text { involvement, } \mathrm{n}(\%)\end{array}$ & $2(1)$ & $0(0)$ & $0(0)$ & $1(1)$ & $1(3)$ \\
\hline Advanced disease stage, $\mathrm{n}(\%)$ & $18(9)$ & 2 (4) & $9(35)$ & $5(5)$ & $2(6)$ \\
\hline Follow-up ended before ERT was available, $n$ (\%) & $8(4)$ & $5(9)$ & $0(0)$ & $3(3)$ & $0(0)$ \\
\hline Other, $\mathrm{n}(\%)$ & $16(8)$ & $2(4)$ & $2(8)$ & $10(10)$ & $2(6)$ \\
\hline
\end{tabular}

${ }^{*}$ Cardiovascular risk factors assessed at first outpatient clinic visit: obesity: Body Mass Index $\geq 30 \mathrm{~kg} / \mathrm{m}^{2}$; smoking: patients who have ever smoked; hypertension: antihypertensive medication use or systolic blood pressure of $>140 \mathrm{~mm} \mathrm{Hg}$ and/or diastolic blood pressure of $>90 \mathrm{~mm} \mathrm{Hg}$, measured at least twice; dyslipidaemia: elevated levels of total cholesterol $(>6.5 \mathrm{mmol} / \mathrm{L})$ or low-density lipoprotein cholesterol $(>2.5 \mathrm{mmol} / \mathrm{L})$ or triglycerides $(>3.0 \mathrm{mmol} / \mathrm{L})$, or low levels of high-density lipoprotein cholesterol (men: $<1.0 \mathrm{mmol} / \mathrm{L}$, women: $<1.2 \mathrm{mmo} / \mathrm{L}$ ) or medication prescribed for the indication dyslipidaemia; diabetes mellitus: type 1 or type 2 , if reported by a medical doctor in the medical chart or when the patient is using antidiabetic medication.

TThe included cardiac MRIs were obtained at the time of the last follow-up (with a maximum range of 2 years between the MRI and the last follow-up date). Severely affected patients with Fabry disease with a non-MRI compatible cardiac device were not included in the routine imaging follow-up.

‡Definition of cardiac hypertrophy on echocardiography: men $>51 \mathrm{~g} / \mathrm{m}^{2.7}$ and women $\left.>48 \mathrm{~g} / \mathrm{m}^{2.7}\right)$, calculated with the Devereux formula: 0.8\{1.04[([LVEDD+IVSd+PWd]3LVEDD3)]\}+0.6. LVEDD, IVSd and PWd are in millimetre.

CVA, cerebrovascular accident; ERT, enzyme replacement therapy; IVSd, interventricular septal thickness at end diastole; LVEDD, left ventricular end diastolic dimension; MR, magnetic resonance; PWd, posterior wall thickness at end diastole.

two cardiologists $(\mathrm{AH}$ and $\mathrm{MB})$ and one metabolic specialist (ML).

\section{Statistical analysis}

For all cardiac events, the event rate per 1000 patient-years was calculated in order to correct for unequal follow-up duration between the different patient groups. The corresponding 95\% CIs were reported for both individual and composite cardiac events, using the mid-P exact test (table 2 and online supplemental table 2). For the event rates calculation, follow-up duration from the age of 15 years onwards was used, because this study shows that cardiac events do not occur before the age of 15 years (figure 2, table 2 and online supplemental table 2) and the event-free follow-up duration of especially the younger men with classical FD would impact unevenly on the event rate without this correction.

Event-free survival (EVS) was analysed using the Kaplan-Meier (KM) method, in which patients where stratified according to phenotype and sex. If less than $50 \%$ of the patients developed a specific event, the median age of onset for those patients who experienced the event was reported instead. Pairwise comparison between patients with FD groups was performed using a logrank test, with Bonferroni correction. Next, these analysis were performed correcting for competing risks (CRs) (eg, non-CVD for the outcome of CVD, online supplemental figures 2-6). Cox regression analyses were performed to assess the effect of phenotype and sex and the interaction between these variables on the 
Table 2 Prevalence, event rates and age at onset of death and cardiovascular events

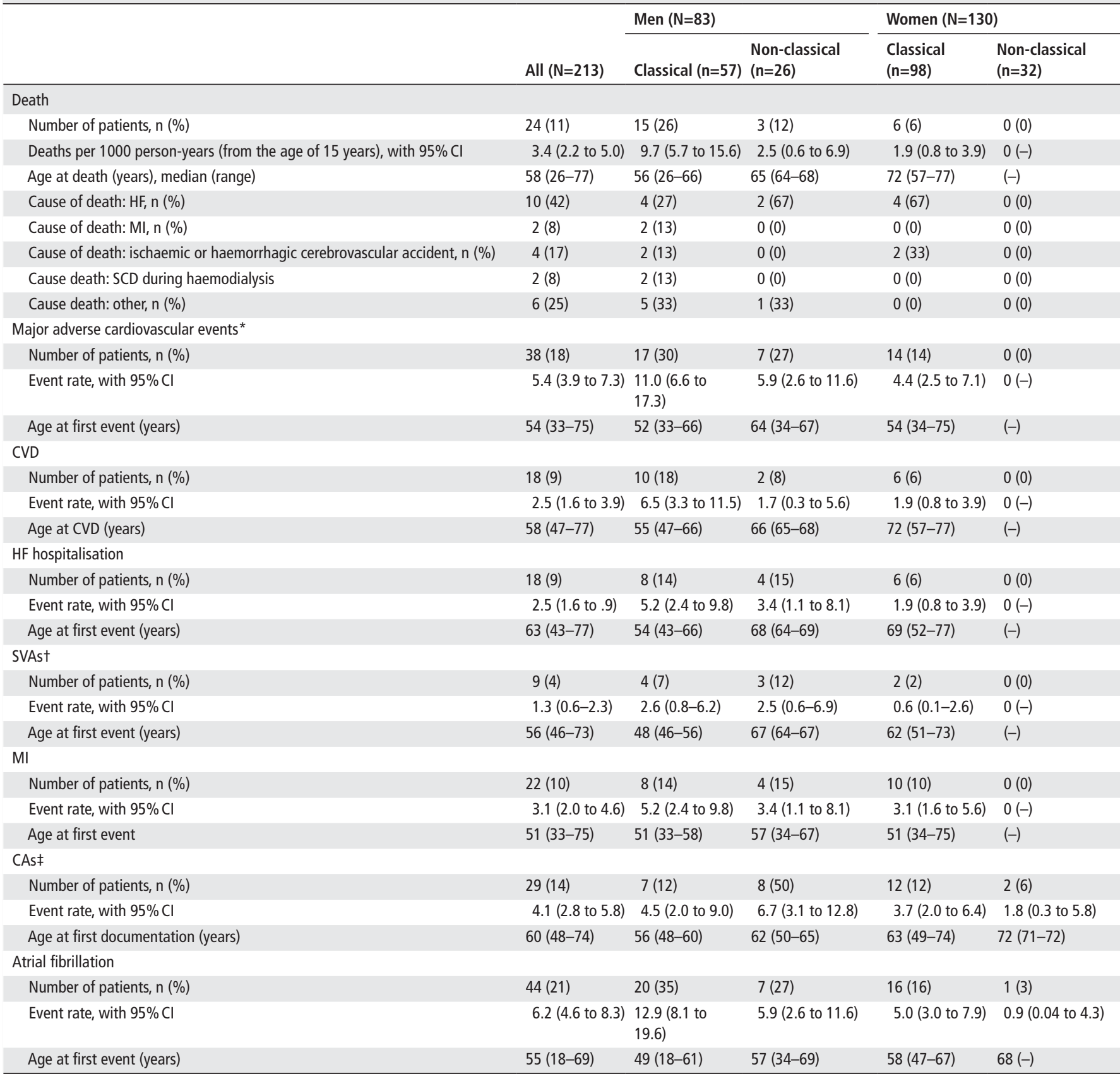

Data are presented as number (percentage) or median (range). All event rates are per 1000 patient-years from the age of 15 years onwards.

* Major adverse cardiac events: composite of CVD, HF hospitalisation, SVAs and MI.

TSVAs: composite of SCD, sudden cardiac arrest, sustained ventricular tachycardia, including appropriate ICD shock, and ventricular fibrillation.

‡CAs: composite of second-degree AV block Mobitz II, third-degree AV block, sinus arrest and pacemaker or ICD device implantation for CAs.

$\mathrm{AV}$, atrioventricular; $\mathrm{CA}$, conduction abnormality; CVD, cardiovascular death; HF, heart failure; ICD, implantable cardiac defibrillator; MI, myocardial infarction; SCD, sudden cardiac death.

occurrence of events. Since no events occurred in the subgroup of women with non-classical FD, we used Firth's penalised Cox regression to obtain stable models. $\mathrm{P}$ values of $<0.008$ (after Bonferroni correction) were considered statistically significant. SPSS V.25 and R V.3.6.1 were used for statistical analysis.

\section{RESULTS}

A total of 213 patients were included: 57 (27\%) men and 98 (46\%) women with classical FD, and $26(12 \%)$ men and $32(15 \%)$ women with non-classical FD. The median age at last outpatient visit or death was 50 years (range 19-83). Patient characteristics are described in table 1 . Figure 2 shows the occurrence of cardiac events for all patients in the four different groups.

\section{Enzyme replacement therapy (ERT)}

Sixty per cent $(128 / 213)$ of the included patients were treated with ERT. Decisions to initiate treatment were based on the presence of symptoms as described earlier ${ }^{25}$ or based on the recommendations of the European Fabry Working Group once they became available. ${ }^{26}$ The majority of the untreated patients were either diagnosed through family screening, without signs of organ involvement at the last follow-up (48\% of untreated 


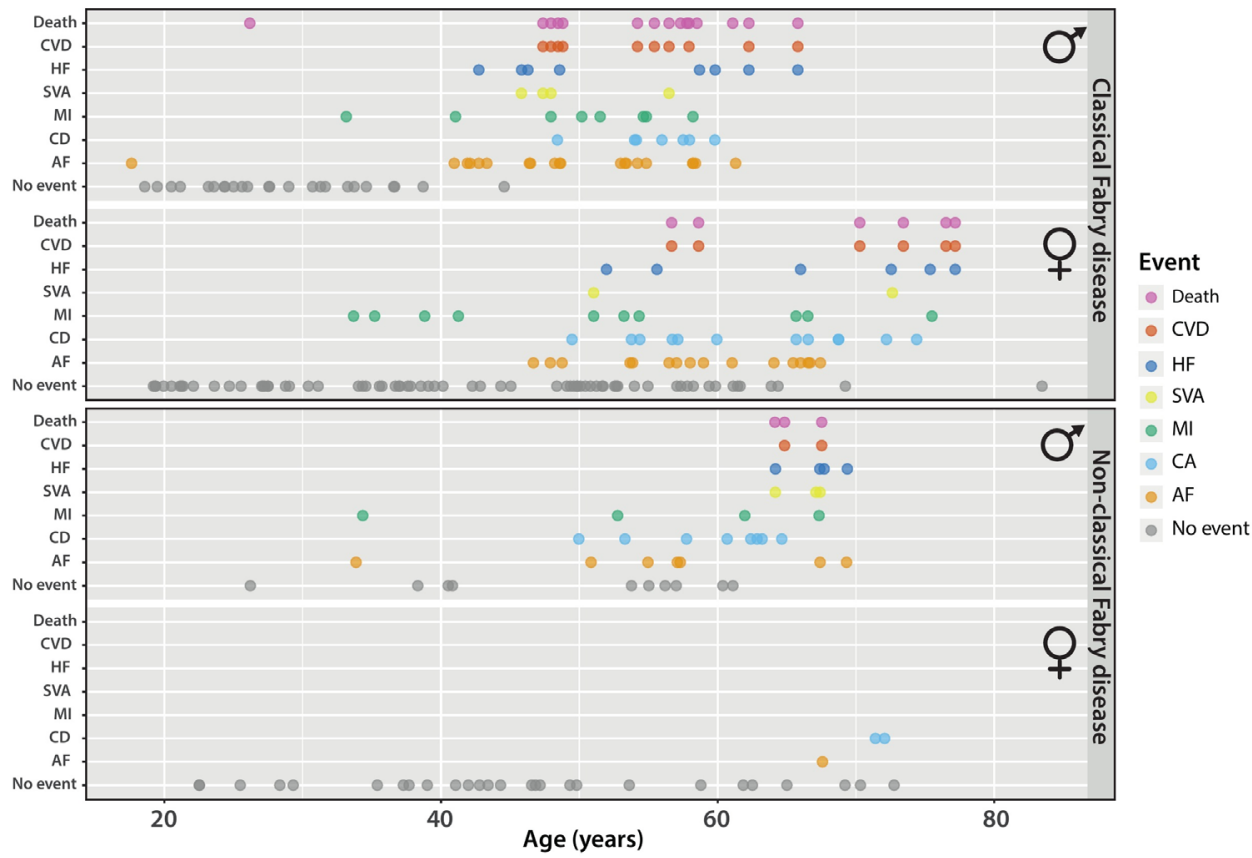

Figure 2 Occurrence of cardiac events for all 213 patients with FD, stratified by sex and phenotype. Included events are death, CVD, HF hospitalisation (first event), SVAs (first event), MI (first event), CA (first recorded) and AF (first recorded). No event was scored if none of the predefined events was recorded at the time of the last outpatient visit. $\hat{O}$, men; $q$, women; $A F$, atrial fibrillation; CA, conduction abnormality; $C V D$, cardiovascular death; HF, heart failure; MI, myocardial infarction; SVA, sustained ventricular arrhythmia.

patients) or diagnosed at an advanced disease stage, at which point no benefit of ERT was to be expected (21\%) (table 1).

\section{Major adverse cardiovascular events}

The event rate (after age 15 years) for MACE was 11.0 per 1000 patient-years (95\% CI 6.6 to 17.3 ) for men with classical FD vs 4.4
(95\% CI 2.5 to 7.1 ) in women with classical FD and 5.9 (95\% CI 2.6 to 11.6 ) in men with non-classical FD. None of the women with non-classical FD developed MACE. KM analysis showed a significant difference between the four subgroups (figure 3; see online supplemental figure 2 for CR analysis). Having a classical phenotype and being male significantly increased the risk of MACE (table 3).

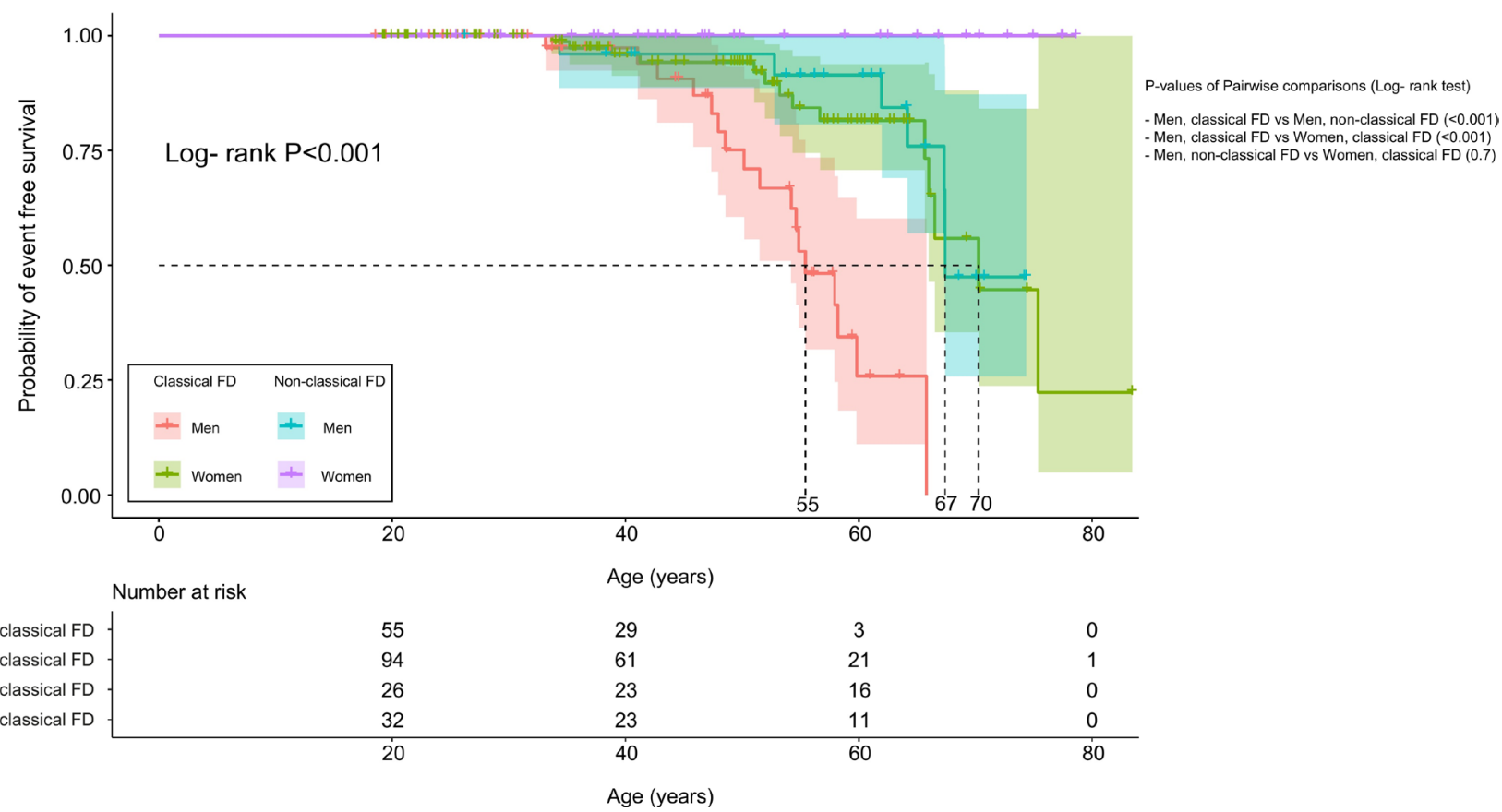

Figure 3 Kaplan-Meier curves (with 95\% Cls) including data of 213 patients with FD, stratified by sex and phenotype, for major adverse cardiovascular events (clustered endpoint of cardiovascular death, heart failure hospitalisation, sustained ventricular arrhythmias and myocardial infarction). Patients are censored if a MACE did not occur at the time of the last follow-up contact. Median event-free survival is given for each group. Pairwise comparisons between patient groups are given. FD, Fabry disease. 


\begin{tabular}{lllll}
\hline Table 3 & \multicolumn{5}{l}{ Firth's penalised Cox regression } \\
\hline Outcome & Covariate & Coef & Exp (coef) & $95 \% \mathrm{Cl}$ \\
\hline MACE & Phenotype, classical & 2.99 & $19.89^{*}$ & 2.61 to 2554.03 \\
& Sex, male & 2.79 & $16.35^{*}$ & 1.94 to 2138.95 \\
& Phenotype:sex & -1.19 & 0.31 & 0.002 to 2.96 \\
CVD & Phenotype, classical & 2.29 & $9.84 \dagger$ & 1.13 to 1292.61 \\
& Sex, male & 1.88 & 6.56 & 0.51 to 916.78 \\
& Phenotype:sex & 0.81 & 2.25 & 0.01 to 38.56 \\
HF & Phenotype, classical & 2.36 & $10.56 \dagger$ & 1.23 to 1383.10 \\
hospitalisation & Sex, male & 2.35 & $10.51 \dagger$ & 1.08 to 1407.18 \\
& Phenotype:sex & 0.38 & 1.47 & 0.01 to 22.14 \\
SVA & Phenotype, classical & 1.07 & 2.91 & 0.23 to 404.25 \\
& Sex, male & 1.81 & 6.14 & 0.58 to 831.73 \\
& Phenotype:sex & 0.55 & 1.74 & 0.01 to 39.94 \\
MI & Phenotype, classical & 2.42 & $11.19 \dagger$ & 1.41 to 1446.63 \\
& Sex, male & 2.21 & 9.12 & 0.94 to 1221.07 \\
& Phenotype:sex & -1.28 & 0.28 & 0.00 to 3.26 \\
\hline${ }^{*}$ P<0.008. & & & & \\
$\dagger P<0.05$. & & & & \\
coeff, coefficient; CVD, cardiovascular death; HF, heart failure; MACE, major \\
adverse cardiovascular event; MI, myocardial infarction; SVA, sustained ventricular \\
arrhythmia.
\end{tabular}

HR for MACE in men with a classical versus non-classical phenotype was 6.1 (95\% CI 2.4-17.0), and that in men vs women with a classical disease phenotype was 5.0 (95\% CI 2.3-11.0) (table 4). Of the patients who developed MACE, MI was the first recorded event in $47 \%$ of men with classical FD, $64 \%$ of women with classical FD and $57 \%$ of men with non-classical FD. Excluding MI from the MACE analysis did not change the observed differences between the patient groups (see online supplemental results).

\section{Cardiovascular death}

In men with classical FD, 10 out of 15 deaths (67\%) were CVDs (event rate 6.5 per 1000 patient years). CVD was also the major cause of death in women with classical FD (6/6 deaths (100\%), event rate 1.9) and men with non-classical FD (2/3 deaths (67\%), event rate 1.7). KM analysis showed a significant difference between the four subgroups (figure 4A; see online supplemental figure 3 for CR analysis). Having a classical phenotype increased the risk of CVD, but this effect did not hold after correcting for multiple testing (table 3). Group comparisons showed significant higher risk in men with classical versus non-classical disease (HR: 22.2, 95\% CI: 5.0-19.3) and men versus women with classical disease (HR: 14.8, 95\% CI: 4.3-67.0) (table 4). HF was an important cause of CVD (40\% of CVD in men with classical FD, $67 \%$ in women with classical FD and 100\% in men with nonclassical FD) (table 2 and online supplemental table 2).

\section{HF hospitalisation}

The event rate for HF hospitalisation was 5.2 in men with classical FD vs 1.9 in women with classical FD. In men with non-classical FD, the event rate was 3.4. KM analysis showed a significant difference between the four subgroups (figure 4B; see online supplemental figure 4 for CR analysis). Classical phenotype and male sex increased the risk of HF hospitalisation, but this effect did not hold after correcting for multiple testing (table 3). Group comparisons showed significant higher risk in men with classical versus non-classical disease (HR: 15.5, 95\% CI: 3.7-82.7) and men versus women with classical disease (HR: 15.4, 95\% CI: 4.1-73.8) (table 4). The left ventricle ejection fraction prior or at the time of the event was known in 17 out of 18 patients, who were hospitalised because of HF. In 12/17 patients $(71 \%)$, the ejection fraction was $<50 \%(5 / 7$ in men with classical FD, 3/6 in women with classical FD and 4/4 in men with non-classical FD).

\section{SVAs and ICD device implantation}

The event rate of SVA was low (14 events in nine patients in the total cohort) (figure 4C; see online supplemental figure 5 for CR analysis; for between-group comparison, see tables 3 and 4). The first SVA episode occurred at the time of an MI in two patients and in one patient at the time of HF hospitalisation. Of the remaining six patients who experienced an SVA, two had an ejection fraction of $<35 \%$; in two patients, the first event occurred during haemodialysis, and in one patient, an allergic reaction to clopidogrel was described as a possible trigger. In the last patient, no trigger could be identified. Of all patients who suffered SVA, three died as a result of the first episode (one due to $\mathrm{MI}$ and two during haemodialysis); one patient died 3 years after the first SVA episode due to MI. The other five patients had an ICD implanted after the first event. In three patients, the ICD successfully aborted an episode of ventricular arrhythmia during follow-up. Of these three patients, two died of HF, respectively, 22 months and 6 years after ICD implantation. One patient was alive at the last follow-up (ICD was 4.8 years in situ). In two patients, no shocks were administered by the ICD. Of these two, one patient died 9 months after ICD implantation as a result of HF (no documented SVA), whereas in the other patient, no shocks were documented at the time of a sudden cardiac arrest with pulseless electrical activity. He was resuscitated and survived the episode. In the entire cohort of 213, 19 patients (including the 5 patients described earlier) had an ICD implanted. The total time the 19 ICDs were in situ was 71 years (range $0.1-9.3$ years per patient).

\section{Myocardial infarction}

MI occurred in 22 patients. MI event rate was 5.2 in men with classical FD, 3.1 in women with classical FD and 3.4 in men with non-classical FD. The median EVS was not significantly different between the three affected patient groups, although the KM curves do show a similar pattern as seen for the other cardiac events (figure 4D and online supplemental figure 6). A classical phenotype increased the risk of MI, but this effect did not hold after correcting for multiple testing (table 3). Group comparisons showed no significant differences in the HRs between the

Table 4 HRs for the comparison of different patient groups

\begin{tabular}{llllcl}
\hline Comparison & MACE & CVD & HF & SVA & MI \\
\hline Men with classical FD versus men with non-classical FD & $6.1(2.4-17.0)^{*}$ & $22.2(5.0-19.3)^{*}$ & $15.5(3.7-82.7)^{*}$ & $5.1(0.9-41.1)$ & $3.1(1.0-11.7)$ \\
\hline Men with classical FD versus women with classical FD & $5.0(2.3-11.0)^{*}$ & $14.8(4.3-67.0)^{*}$ & $15.4(4.1-73.8)^{*}$ & $10.7(1.9-92.0)^{*}$ & $2.5(1.0-6.7)$ \\
Men with non-classical FD versus women with classical FD & $0.8(0.3-2.0)$ & $0.7(0.1-2.7)$ & $1(0.3-3.4)$ & $2.1(0.4-13.0)$ & $0.8(0.2-2.4)$ \\
\hline
\end{tabular}

These HRs where obtained from Firth's penalised COX regression models in which sex and phenotype where combined in one variable with four patient groups.

${ }^{*} P<0.008$.

CVD, cardiovascular death; FD, Fabry disease; HF, heart failure; MACE, major adverse cardiovascular event; MI, myocardial infarction; SVA, sustained ventricular arrhythmia. 
P-values of Pairwise comparisons (Log- rank test)

- Men, classical FD vs Men, non-classical FD $(<0.001)$

- Men, classical FD vs Women, classical FD $(<0.001)$

- Men, non-classical FD vs Women, classical FD (0.5)

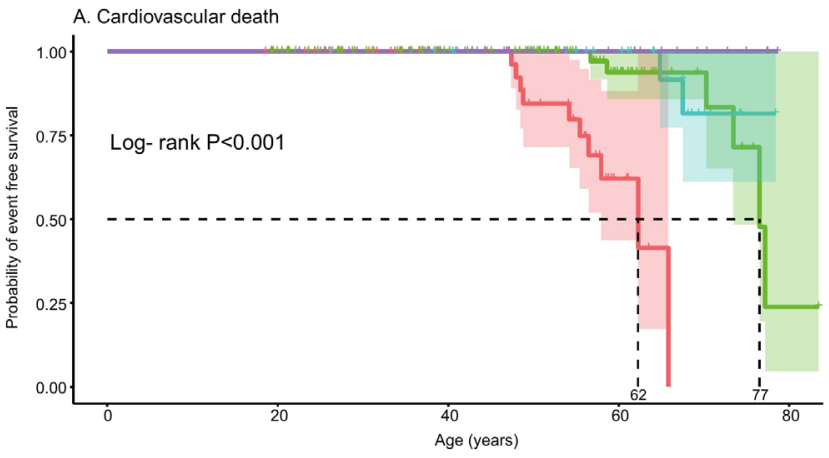

C. Sustained ventricular arrhythmias

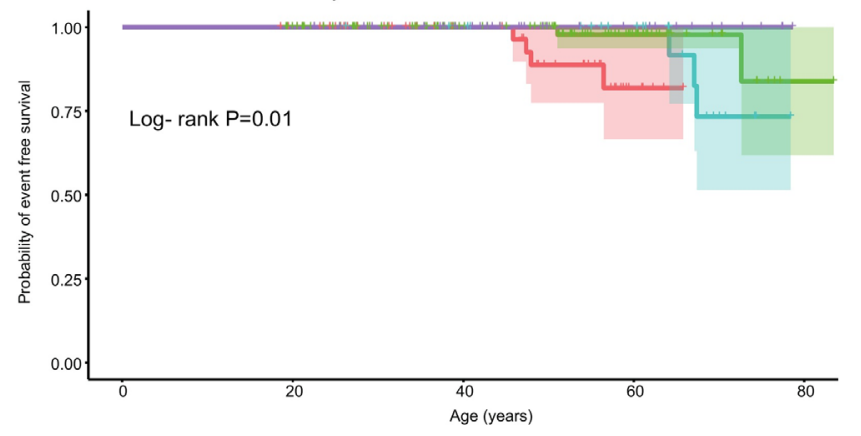

P-values of Pairwise comparisons (Log- rank test) - Men, classical FD vs Men, non-classical FD (0.06 - Men, classical FD vs Women, classical FD (0.01)

- Men, non-classical FD vs Women, classical FD (0.4)
P-values of Pairwise comparisons (Log- rank test)

- Men, classical FD vs Men, non-classical FD $(<0.001)$

Men, classical FD vs Women, classical FD $(<0.001)$

- Men, non-classical FD vs Women, classical FD $(1,0)$

B. Heart failure hospitalization

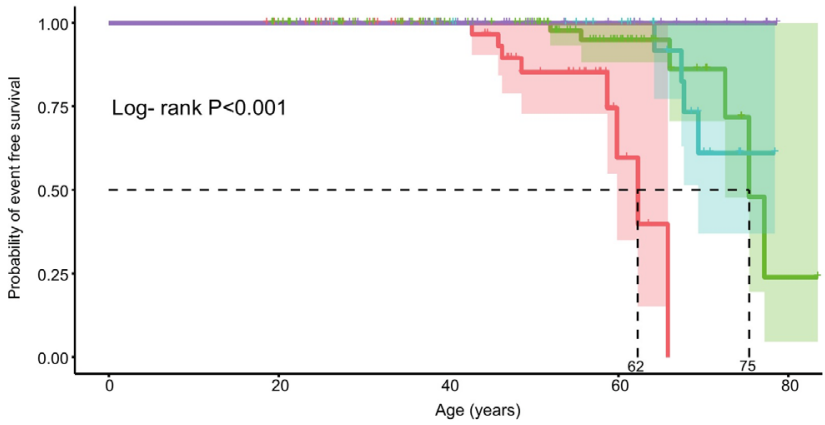

D. Myocardial infarction

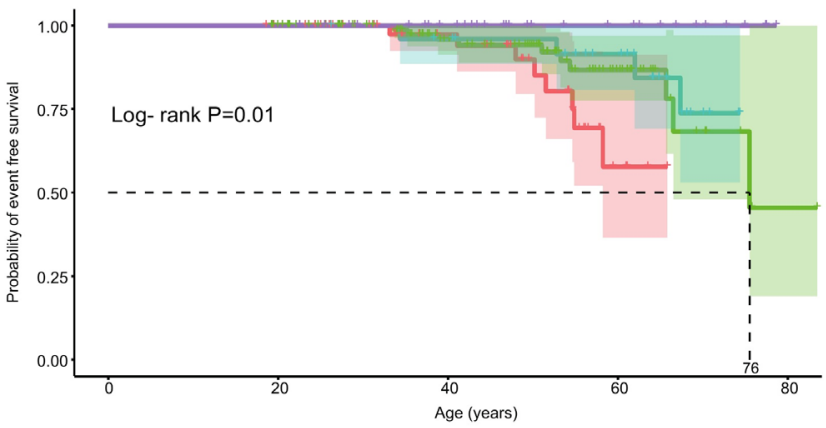

$\mathrm{P}$-values of Painwise comparisons (Log- rank test)

- Men, classical FD vs Men, non-classical FD (0.05)

- Men, classical FD vs Women, classical FD (0.06)

- Men, non-classical FD vs Women, classical FD (0.7)

Figure 4 Kaplan-Meier curves (with 95\% Cls) including data of 213 patients with FD, stratified by sex and phenotype for (A) cardiovascular death, (B) heart failure hospitalisation, (C) sustained ventricular arrhythmias and (D) myocardial infarction. Patients are censored if the event did not occur at the time of the last follow-up contact. Median event-free survival is given for each group in which events occurred. Pairwise comparisons between patient groups are given for each cardiac outcome. FD, Fabry disease.

patient groups (table 4; for further MI classification, see online supplemental table 3). Cardiovascular risk factors (obesity, smoking, hypertension, dyslipidaemia and diabetes mellitus) in patients suffering from an MI were present in 4 out of 8 men with classical FD (data not available for two patients), in 8 out of 10 women with classical FD and in 4 out of 4 men with nonclassical FD (data not shown).

Data on other non-major cardiac events can be found in the online supplemental results section.

\section{DISCUSSION}

This is the first longitudinal study that describes the prevalence and timing of cardiac events in a large cohort of male and female patients with FD, categorised into classical and non-classical patient groups. The results show that these sex-defined and phenotype-defined patient groups differ substantially in terms of their risk of major cardiac events. The risk for all events showed the same trend: highest in men with a classical FD, intermediate in women with classical FD and in men with non-classical FD, and low in women with non-classical FD. For the individual events, differences between the groups were not always significant due to the relative low event rate. In men with classical FD, events occurred mainly from the fifth decade of life onwards, resulting in a reduced life expectancy (no men with classical FD in our cohort survived beyond 66 years of age). In women with classical FD and men with non-classical FD, events are observed from the sixth decade onwards, with more variability regarding the age of onset. MI is the first major adverse cardiac event observed in 21/38 (55\%) of patients with a MACE. Most likely, both macrovascular and microvascular CADs contribute to the development of ischaemia due to endothelial dysfunction and cardiac hypertrophy in FD. ${ }^{17} 2728$ The relative contribution of FD-related endothelial pathology and hypertrophy versus that of general risk factors (eg, smoking) cannot easily be determined, given that the majority of patients had cardiovascular risk factors at the time of MI.

Importantly, none of the women with non-classical FD developed a MACE. This variation means that if 'cardiac events' is a clinical endpoint in an FD treatment study, men with classical FD should be treated as a separate group from women with classical FD and men with non-classical FD, and women with non-classical FD should not be included at all. This also means that results from previous clinical trials, in which phenotypical distinction was not made, should be interpreted with caution.

Fabry cardiomyopathy is associated with a risk of SCD, and in advanced disease, ICD implantation should be considered. ${ }^{15} 29$ The most frequently observed cause of death in the current study was HF (42\% of all deaths), whereas SCD comprised $17 \%$ of all deaths. This finding is in contrast with a recent meta-analysis of Baig et al, which identified SCD as the most common cause of 
death (62\% of all deaths). However, the context of SCD and the FD phenotype of the included patients were not clear for most studies included in this meta-analysis. In addition, most included studies were performed in small cohorts, with a relatively short follow-up duration. In our patients with FD cohort, the SVA rate was low and more than half of the patients developed their first event in the context of either an MI or HF (hospitalisation or ejection fraction<35\%). An earlier study of Vijapurapu et $a l^{29}$ reported a higher event rate in patients with FD with an ICD, but sex and phenotype distributions in this group were not reported. Large multicentre cohort studies are needed to develop an FD SCD risk calculator to guide ICD implantation decision making.

Our findings, if confirmed in other longitudinal cohort studies, may change decision making regarding ICD implantation policy in FD. With the knowledge that HF is the main contributor to death in FD, future studies should aim to detect and treat HF in a much earlier phase of the disease. This highlights the urgent need for biomarkers that predict future development of HF, separating low-risk and high-risk patients, as clearly not all women with classical FD and men with non-classical FD develop HF.

A limitation of the current study is its retrospective design, which may lead to under-recording and subsequent underestimation of the event risk, even though we accessed historical files for the majority of patients. Nevertheless, we cannot rule out that for some deceased patients, especially those who died over a decade ago, some cardiac events may have been missed. In addition, the reported age of onset of cardiac manifestations such as

\section{Key messages}

\section{What is already known on this subject?}

- Early cardiac manifestations of Fabry disease (FD) are left ventricular hypertrophy, fibrosis formation and conduction abnormalities. As the disease progresses, cardiac complications, such as arrhythmias and heart failure (HF), occur in a significant proportion of patients with $F D$, yet others remain asymptomatic throughout long-term followup. The evolution of cardiac events and the influence of sex and disease phenotypes on this trajectory have not been described previously.

\section{What might this study add?}

- This is the first large longitudinal cohort study describing the effect of disease phenotype and sex on the occurrence of cardiac events in FD. The event rates of major adverse cardiovascular events (combined endpoint cardiovascular death (CVD), HF hospitalisation, sustained ventricular arrhythmias (SVA) and myocardial infarction) were 11.0 in men with classical $\mathrm{FD}(95 \% \mathrm{Cl} 6.6$ to 17.3$)$ (event-free survival (EVS) 55 years), $4.4(95 \% \mathrm{Cl} 2.5$ to 7.1$)$ and in women with classical FD (EVS 70 years) and 5.9 (2.6-11.6) in men with non-classical FD (EVS 70 years). None of these events occurred in women with non-classical FD. CVD was the leading cause of death $(75 \%)$, to which $\mathrm{HF}$ contributed most $(42 \%)$. The overall rate of SVAs was low (14 events in nine patients $(4 \%))$

\section{How might this impact on clinical practice?}

- Cardiac care in FD should be tailored to the sex and disease phenotypes of the patient and should focus on early detection and treatment of HF. systolic dysfunction, LVOT obstruction and heart valve disease depends on the time at which imaging was performed and may thus not be fully accurate. The contribution of enrolment bias on the observed outcomes between sexes remains unknown. In theory, more women could have been identified through family screening and more men because of clinical manifestations, which could have contributed to the observed differences in the occurrence of cardiac events. The influence of chronic kidney disease on MACE occurrence, which may have increased the risk, was not analysed as the sample size and differences in follow-up duration between patients did not allow for this analysis. However, a glomerular filtration rate below $60 \mathrm{~mL} / \mathrm{min}$ was present only in $20 \%$ of classically affected male patients and only in a single patient from other groups (online supplemental table 4). In addition, the effect of ERT on the occurrence of cardiac events was not analysed, because of the same reasons, as well as variability in the age of therapy initiation and indication bias for start of treatment.

An important strength of the study is the fact that it was a long-term longitudinal study on a large FD cohort, and the detailed data on predefined clinical cardiac events, in contrast to earlier studies, which often used composites for cardiac involvement in FD, including symptoms of angina pectoris, palpitations and microscopic $\mathrm{Gb} 3$ storage. ${ }^{22} 23$

\section{CONCLUSIONS}

This large longitudinal study confirms that men with classical FD develop severe cardiac events, mainly from the fifth decade of life onwards. For women with classical FD and men with non-classical FD, cardiac events occur approximately a decade later and in a smaller proportion of patients. None of the non-classically affected women in this cohort developed a major cardiac event. More than half of the first observed SVAs occurred in the context of either an MI or HF, and HF was the most common cause of death. These findings shed new light on the clinical course and cardiac outcomes in FD cardiomyopathy and emphasise the need for new treatments, primarily to prevent $\mathrm{HF}$ in FD.

Acknowledgements We thank Dr. Alexander Maass (University Medical Center Groningen, Cardiologist) and Dr Janneke Timmermans (Radboud University Medical Center Nijmegen, Cardiologist), Elke de Koning (Amsterdam University Medical Center, doctor's assistant) and Lydia Veerhuis (Amsterdam University Medical Center, metabolic diseases coordinator) for their contribution in collecting the data. The authors thank Sanne van der Veen for her help in the graphical design.

Contributors MES: study design, acquisition, analysis and interpretation of data, first draft of manuscript. AH: study design, interpretation of data, critical revision of manuscript. MB: study design, interpretation of data, critical revision of manuscript. LvD: analysis and interpretation of data, critical revision of manuscript. MD: data extraction from electronic clinical report files, data processing, critical revision of manuscript. $\mathrm{CH}$ : study concept, study design, interpretation of data and critical revision of manuscript. ML: study concept, study design, study supervision, interpretation of data and critical revision of manuscript.

Funding This work was supported by SPHINX, the Amsterdam Lysosome Center.

Disclaimer MES is as a subinvestigator involved in a premarketing study with Idorsia. Financial arrangements were made through AMC Research BV. No fees, travel support or grants were obtained from Pharmaceutical Industry. No support or grants were accepted in relation to the submitted work. ML and $\mathrm{CH}$ are involved in premarketing studies with Genzyme, Protalix and Idorsia.

\section{Competing interests None declared.}

\section{Patient consent for publication Not required.}

Ethics approval The medical ethics review committee of the Amsterdam University Medical Centres confirmed that the Medical Research Involving Human Subjects Act does not apply to this study (W19-438 \# 19.505) because of the retrospective character of this work, using historical data obtained in the context of 
regular patient care. The study was conducted in accordance with the Declaration of Helsinki.

Provenance and peer review Not commissioned; externally peer reviewed. Data availability statement Data are available upon reasonable request.

Supplemental material This content has been supplied by the author(s). It has not been vetted by BMJ Publishing Group Limited (BMJ) and may not have been peer-reviewed. Any opinions or recommendations discussed are solely those of the author(s) and are not endorsed by BMJ. BMJ disclaims all liability and responsibility arising from any reliance placed on the content. Where the content includes any translated material, BMJ does not warrant the accuracy and reliability of the translations (including but not limited to local regulations, clinical guidelines, terminology, drug names and drug dosages), and is not responsible for any error and/or omissions arising from translation and adaptation or otherwise.

Open access This is an open access article distributed in accordance with the Creative Commons Attribution Non Commercial (CC BY-NC 4.0) license, which permits others to distribute, remix, adapt, build upon this work non-commercially, and license their derivative works on different terms, provided the original work is properly cited, appropriate credit is given, any changes made indicated, and the use is non-commercial. See: http://creativecommons.org/licenses/by-nc/4.0/.

\section{ORCID iDs}

Mohamed El Sayed http://orcid.org/0000-0003-4620-6162

Alexander Hirsch http://orcid.org/0000-0002-3315-2006

\section{REFERENCES}

1 Brady ROet al. Enzymatic Defect in Fabry's Disease 1967;276:1163-7.

2 Kint JA. Fabry's Disease: Alpha-Galactosidase Deficiency 1970;167:1268-9.

3 Mehta A, Clarke JTR, Giugliani R, et al. Natural course of Fabry disease: changing pattern of causes of death in FOS - Fabry Outcome Survey. J Med Genet 2009;46:548-52.

4 Germain DP. Fabry disease. Orphanet J Rare Dis 2010;5:30.

5 Mehta A, Ricci R, Widmer U, et al. Fabry disease defined: baseline clinical manifestations of 366 patients in the Fabry outcome survey. Eur J Clin Invest 2004:34:236-42.

6 Smid BE, van der Tol L, Biegstraaten M, et al. Plasma globotriaosylsphingosine in relation to phenotypes of Fabry disease. J Med Genet 2015;52:262-8.

7 Arends M, Wanner C, Hughes D, et al. Characterization of classical and nonclassical Fabry disease: a multicenter study. J Am Soc Nephrol 2017;28:1631-41.

8 von Scheidt W, Eng CM, Fitzmaurice TF, et al. An atypical variant of Fabry's disease with manifestations confined to the myocardium. N Engl J Med 1991:324:395-9.

9 Nakao S, Takenaka T, Maeda M, et al. An atypical variant of Fabry's disease in men with left ventricular hypertrophy. N Engl J Med 1995;333:288-93.

10 Sachdev B, Takenaka T, Teraguchi $\mathrm{H}$, et al. Prevalence of Anderson-Fabry disease in male patients with late onset hypertrophic cardiomyopathy. Circulation 2002:105:1407-11.
11 Nordin S, Kozor R, Medina-Menacho K, et al. Proposed Stages of Myocardial Phenotype Development in Fabry Disease. JACC Cardiovasc Imaging 2019;12:1673-83.

12 Nordin S, Kozor R, Baig S, et al. Cardiac phenotype of prehypertrophic Fabry disease. Circ Cardiovasc Imaging 2018;11:e007168.

13 Wilson HC, Hopkin RJ, Madueme PC, et al. Arrhythmia and clinical cardiac findings in children with Anderson-Fabry disease. Am J Cardiol 2017;120:251-5.

14 Weidemann F, Maier SKG, Störk S, et al. Usefulness of an implantable loop recorder to detect clinically relevant arrhythmias in patients with advanced Fabry cardiomyopathy. Am J Cardiol 2016:118:264-74.

15 Baig Set al. Ventricular arrhythmia and sudden cardiac death in Fabry disease: a systematic review of risk factors in clinical practice. Europace 2017.

16 Frustaci A, Russo MA, Francone $M$, et al. Microvascular angina as prehypertrophic presentation of Fabry disease cardiomyopathy. Circulation 2014;130:1530-1.

17 Chimenti C, Morgante E, Tanzilli G, et al. Angina in Fabry disease reflects coronary small vessel disease. Circulation 2008;1:161-9.

18 Liu D, Oder D, Salinger T, et al. Association and diagnostic utility of diastolic dysfunction and myocardial fibrosis in patients with Fabry disease. Open Heart 2018:5:e000803.

19 Sené T, Lidove 0, Sebbah J, et al. Cardiac device implantation in Fabry disease: a retrospective monocentric study. Medicine 2016:95:e4996.

20 Bodary PF, Shayman JA, Eitzman DT. Alpha-Galactosidase A in vascular disease. Trends Cardiovasc Med 2007:17:129-33.

21 Schiffmann R, Rapkiewicz A, Abu-Asab M, et al. Pathological findings in a patient with Fabry disease who died after 2.5 years of enzyme replacement. Virchows Arch 2006;448:337-43.

22 Linhart A, Kampmann C, Zamorano JL, et al. Cardiac manifestations of AndersonFabry disease: results from the International Fabry outcome survey. Eur Heart J 2007;28:1228-35.

23 Favalli V, Disabella E, Molinaro M, et al. Genetic screening of Anderson-Fabry disease in probands referred from Multispecialty clinics. J Am Coll Cardiol 2016:68:1037-50.

24 Smid BE, van der Tol L, Cecchi F, et al. Uncertain diagnosis of Fabry disease: consensus recommendation on diagnosis in adults with left ventricular hypertrophy and genetic variants of unknown significance. Int J Cardio/ 2014;177:400-8.

25 Vedder AC, Linthorst GE, Houge G, et al. Treatment of Fabry disease: outcome of a comparative trial with agalsidase alfa or beta at a dose of $0.2 \mathrm{mg} / \mathrm{kg}$. PLoS One 2007;2:e598.

26 Biegstraaten M, Arngrímsson R, Barbey F, et al. Recommendations for initiation and cessation of enzyme replacement therapy in patients with Fabry disease: the European Fabry Working group consensus document. Orphanet J Rare Dis 2015;10:36.

27 Tomberli B, Cecchi F, Sciagrà R, et al. Coronary microvascular dysfunction is an early feature of cardiac involvement in patients with Anderson-Fabry disease. Eur J Heart Fail 2013;15:1363-73.

28 Kitani Y, Nakagawa N, Sakamoto N, et al. Unexpectedly high prevalence of coronary spastic angina in patients with Anderson-Fabry disease. Circ J 2019;83:481-4.

29 Vijapurapu R, Geberhiwot T, Jovanovic A, et al. Study of indications for cardiac device implantation and utilisation in Fabry cardiomyopathy. Heart 2019;105:1825-31. 